\section{Improving AZT efficacy}

To the editor-In the August 1997 issue of Nature Medicine, Lavie et al. describe the molecular and pharmacological basis of the bottleneck in AZT activation'. They suggest that a more efficient conversion of AZT-monophosphate to AZT-triphosphate would result in a higher antiviral efficacy, lower toxicity and a reduced emergence of AZT-resistant virus strains, and propose a gene therapy approach with mutant yeast or human thymidylate (TMP) kinase that has an improved capacity to phosphorylate AZT-MP to its triphosphate. It is not clear how to accomplish such a task by simple site-directed mutagenesis of the enzyme and instead we propose the murine wild-type thymidylate kinase as the enzyme of choice to improve AZT-MP phosphorylation since murine cells (eg. leukemia L1210, breast carcinoma FM3A and embryo fibroblast L929) do not accumulate AZT-MP and are better able to convert AZT-MP to AZT-TP than are human cells (ref. 2 and unpublished data).

However, other considerations should also be taken into account. In human cells, the rate-limiting conversion of AZTMP to AZT-DP not only results in AZT-MP accumulation but is also accompanied by TMP accumulation due to inhibition of the TMP kinase by AZT-MP ${ }^{3,4}$. Thus lowering intracellular AZT-MP levels will not only be accompanied by an enhanced phosphorylation of AZT-MP to AZT-DP and AZT-TP, but will also lead to enhanced formation of TDP and TTP. The increased TTP levels may, in turn, diminish the inhibitory effects of AZT-TP on HIV reverse transcriptase (RT) since AZTTP needs to compete with TTP to inhibit HIV RT. In addition, TTP allosterically inhibits thymidine kinase (TK) activity, and thus feedback inhibition of the first activation step of AZT (by the cytosolic TK) by the increased TTP pool levels, may indirectly suppress AZT-TP formation. Moreover, we found that AZT-TP is also a potent allosteric inhibitor of cytosolic TK activity at physiological concentrations (i.e. lower micromolar range) (unpublished observations). Thus, increasing both intracellular TTP and AZT-TP levels will clearly have a feed-back suppressive effect on the eventual conversion of AZT to AZT-TP.

The observation that yeast TMP kinase and human TMP kinase have $42.9 \%$ sequence identity, and yeast TMP kinase and murine TMP kinase have $43.6 \%$ sequence identity including the same highly conserved amino acids that are assumed to play a role in the catalysis of the TMP kinase reaction, may make a site-directed mutagenesis approach difficult. Therefore, it is too optimistic to expect a marked improvement of the antiviral properties of AZT by introducing mutated human TMP kinase into (HIV-infected) cells. Instead, expression of murine TMP kinase in human cells will provide greater insight in the eventual feasibility of the gene therapy approach to improve the anti-HIV activity of AZT.

\section{J. Balzarini, B. Degrève}

\& E. DE ClercQ

Rega Institute for Medical Research

K.U.Leuven

Minderbroedersstraat 10

B-3000 Leuven, Belgium

jan.balzarini@rega.kuleuven.ac.be

Lavie and colleagues reply-The objective of our approach is to render the cells (or more exactly the reverse transcriptase (RT)-dependent HIV-replication) more susceptible to $3^{\prime}$-deoxy-3'-azido thymidine (AZT). Despite criticizing this suggestion as being naive, Balzarini et al. also consider the idea worth testing. They suggest the use of murine thymidylate kinase, since cell culture experiments have shown that AZT is converted more efficiently to its triphosphate form in murine than in human cells ${ }^{5}$. However, we would like to poir : out that there are two different form of murine thymidylate kinase, with significantly different sequences and, presumably, structural characteristics. Unfortunately, the cell culture results reported by Balzarini et al. have not been substantiated by experiments on isolated or recombinant proteins. As such, we do not know to which form of the enzyme the more efficient phosphorylation of AZTMP should be attributed, or whether TmpK is really responsible for the effects reported. Until these data have been obtained it is difficult to discuss the relative properties of the human and murine enzymes with respect to AZT-MP.

Turning to the specific points raised, it is true that increasing thymidylate kinase activity for AZT-MP may also increase the amount of dTTP formed. Nevertheless, increasing the rate of phosphorylation of
AZT-MP relative to dTMP will tend to increase the relative ratio of AZT-TP to dTTP, which is the important parameter in determining the degree of inhibition of RT. The allosteric feedback inhibition of thymidine kinase by dTTP and AZT-TP will have similar effects on both AZT-MP and dTMP production. An interesting possibility is to combine the gene transfer approach suggested with the use of a masked neutral AZT-MP derivative that is able to penetrate membranes ${ }^{6}$. Thus, if high AZT-TP concentrations inhibit thymidine kinase, dTMP production will be inhibited, while the conversion of a masked AZT-MP prodrug to AZT-MP will not be affected, again tending to increase the AZT-TP/dTTP ratio.

We agree that any enzyme capable of phosphorylating AZT-MP efficiently to its diphosphate form is of interest and may be useful for gene therapy and in fact we have now identified such a kinase ${ }^{7}$. However we believe that there are immunological and other advantages to using a mutant human enzyme.

ARnON LAVIE, Jochen ReINSTEIN, ROGER S. GoOdy \& ILME SCHLICHTING Max-Planck-Institute for Molecular Physiology Department of Physical Biochemistry Rheinlanddamm 201

44139 Dortmund, Germany

MANFred KonRad

Max-Planck-Institute for Biophysical Chemistry Department of Molecular Genetics

37018 Göttingen, Germany

1. Lavie, A. et al. The bottleneck in AZT activation Nature Med. 3, 922-924 (1997).

2. Balzarini, 1 . et al. The in vitro and in vivo anti-retrovirus activity, and intracellular metabolism of $3^{\prime}$ azido-2', $3^{\prime}$-dideoxythymidine and 2', $3^{\prime}$-dideoxycytidine are highly dependent on the cell species. Biochem. Pharmacol. 37, 897-903 (1988).

3. Balzarini, J., Herdewijn, P. \& De Clercq, E. Differential patterns of intracellular metabolism of $2^{\prime}, 3^{\prime}$-didehydro-2', $3^{\prime}$-dideoxythymidine and $3^{\prime}$ azido-2', 3'-dideoxythymidine, two potent antihuman immunodeficiency virus compounds. J. Biol. Chem. 264, 6127-6133 (1989)

4. Frick, L.W. et al. Effects of 3'-azido-3'-deoxythymidine on the deoxynucleotide triphosphate pools of cultured human cells. Biochem. Biophys. Res. Comm. 154, 124-129 (1988).

5. Guettari, N., Loubiere, L., Brisson, E. \& Klatzmann, D. Use of Herpes-simplex Virus Thymidine Kinase to Imporve the Antiviral Activity of Zidovudine. Virology 235, 398-405 (1997).

6. Meier, C., Habel, L., Laux, W., Declercq, E., \& Balzarini, J. Homo Dinucleoside-alpha-hydroxyphosphonate Diesters as Prodrugs of the Antiviral Nucleoside Analogues $2^{\prime}, 3^{\prime}$-Dideoxythymidine and 3'-Azido-2', 3'Dideoxythymidine. Nucleosides \& Nucleotides 14, 759-762 (1995).

7. Lavie, A et al. Crystal Structure of Yeast Thymidylate Kinase Complexed with the Bisubstrate Inhibitor P1-(5'-Adenosyl)P5-(5'-Thymidyl)Pentaphosphate $\left(T_{5} A\right)$ at $2.0 \AA$ Resolution: Implications for Catalysis and AZT Activation Biochem. In the press. 